\title{
Use of Peripheral Health Units in Low-Transmission Ebola Virus Disease Surveillance
}

\author{
Alyssa J. Young ${ }^{*}$, Allison Connolly, Adam Hoar, Brooke Mancuso, John Mark Esplana, \\ Guddu Kaur, Laura Fisher, Mary-Anne Hartley and Anh-Minh A Tran
}

GOAL Global, Dublin, Ireland

\section{Objective}

A community-based EVD surveillance system with improved symptom recording and follow-up of malaria positive patients at PHUs was implemented during low EVD transmission. The rationale and methodology in implementing a PHU-focused approach to strengthen surveillance system sensitivity is described.

\section{Introduction}

Existing EVD surveillance strategies in Sierra Leone use a centralized live alert system to refer suspect cases from the community to Ebola treatment centers. As EVD case burden declined in Port Loko District, so did the number of reported alerts. As EVD presents similarly to malaria, the number of alerts reported are expected to remain consistent with malaria prevalence in malaria-endemic areas, irrespective of a reduction in true EVD cases. Declines in reported suspect cases from the community alluded to the possibility that individuals were returning to healthcare centers to seek treatment for malaria, and that PHUs were not adequately reporting suspect EVD cases. District surveillance officers (DSOs) were used to investigate the usage of PHUs by community members, as well as the mechanisms that health center staff used in recording patient visits. Surveillance methods specific to PHUs were introduced to increase the number of reported EVD alerts, as well as establish the foundation for future integrated disease surveillance response strategies.

\section{Methods}

PHU surveillance methodology focused on 4 primary components:

- Initial Evaluation: PHU-specific evaluations were conducted to gauge the level of community use of primary health care structures as EVD case-load decreased. "Under-five", OPD, and Triage registers were reviewed to investigate what disease recording mechanisms were used by healthcare staff.

- Case Definition Education: healthcare staff were educated on EVD case definition and protocol for reporting all suspect cases through the centralized live alert system.

-Improved Symptom Recording: a section in all patient registries was included specifically for recording symptoms of any patient upon arrival at a PHU. Recording of patient symptoms, as opposed to only presumed diagnosis, was introduced as a tool for surveillance of a greater range of diseases by allowing for easy review of demographicspecific trends in symptomatic presentations. Recording symptoms of individuals with a positive rapid diagnostic test (RDT) not only forms the foundation for a malaria patient follow-up system but also allows symptomatic groupings for differential EVD diagnosis.

- Malaria algorithm: a system was introduced to aid healthcare staff in eliminating possibilities of malaria and EVD co-infection among patients presenting at PHUs. As arteminisin combination therapy (ACT) is fast acting and can reduce parasitemia levels within 1-2 days, an algorithm was proposed for improved surveillance (fig. 1.)

\section{Results}

Initial PHU evaluations showed that the majority of community members weren't actually returning to PHUs, and that the decrease in live alerts was a result of poor community reporting, unrelated to receipt of healthcare at PHUs. As a result, social mobilization and community engagement efforts were adapted to stress the importance of returning PHUs for primary healthcare needs. The PHU EVD surveillance system was used to implement several small, but effective changes to the recording of patient symptoms, follow-up of malaria confirmed patients, and contributed to an increase in communityreported live and death alerts in the district (fig. 2).

\section{Conclusions}

PHU attendance was gauged as an indicator of baseline disease in order to investigate the decrease in live alerts in the EVD reporting system. Low reporting could be correlated to low PHU attendance and indicated a need for social mobilization and community engagement.

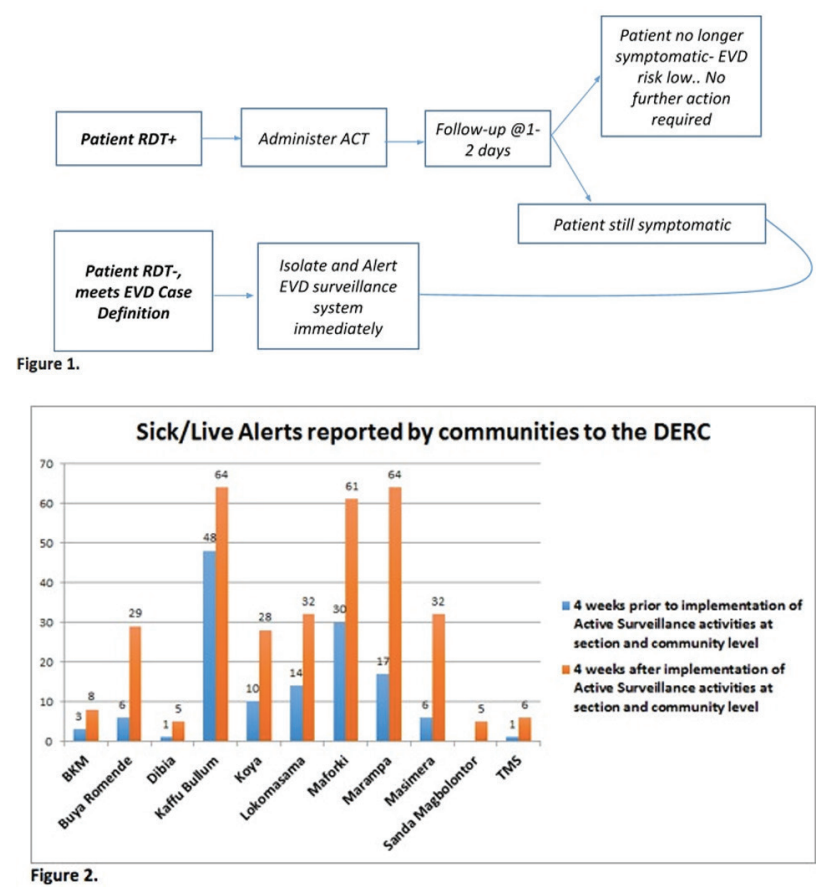

Keywords

Ebola Virus Disease surveillance; Peripheral health unit; Sierra Leone; malaria

\section{Acknowledgments}

GOAL would like to acknowledge the contributions of the Port Loko District Health Management Team, District Ebola Response Center, WHO, and CDC

\section{*Alyssa J. Young}

E-mail: ayoung@sl.goal.ie 